\title{
The Use of Human 3D Reconstructed Airway Cultures for Tobacco Product Evaluation: Precision Low-Volume Exposures at the Apical Site
}

\author{
Holger P. Behrsing, ${ }^{1}$ Song Huang, ${ }^{2}$ and Samuel Constant ${ }^{2}$
}

\begin{abstract}
With a mandate to evaluate the dynamics of pulmonary exposure to inhaled materials such as tobacco-based products, researchers are employing complex, human, three-dimensional pulmonary models. Human reconstructed airway (RHuA) tissues present a platform that more closely resembles airways in vivo. Grown at the air-liquid interface (ALI), RHuA tissues offer apical and basal compartments that allow flexibility in modeling physiologically relevant exposures and provide sampling location-specific results. Various instruments can produce smoke/aerosols (including that from e-cigarette) and expose tissues at the apical surface, but the quantitation of materials deposited remains a challenge. Alternatively, a solution containing solubilized materials can be applied through a pipette, but this less physiologically relevant method may adversely impact the tissue. We have tested the HP D300 digital dispenser as a means to deliver patterned picoliter amounts of dimethyl sulfoxide-based material (including total particulate matter) onto Epithelix MucilAir ${ }^{\mathrm{TM}}$ tissues. Release markers, viability assessment, and ciliary beat frequency $(\mathrm{CBF})$ were compared in both the apical and basolateral compartments after 72 hours of exposure. Results of this work demonstrated pattern and volume dispensing accuracy. With the exception of $\mathrm{CBF}$, no significant adverse effect from up to $707 \mathrm{~nL}$ total single dispense volume was detected using release marker or viability assays. This novel technology has demonstrated promising results as a method by which precise amounts of solubilized materials (e.g., tobacco-based extracts) may be delivered onto the apical surface of tissue grown at the ALI.
\end{abstract}

Keywords: 3D airway exposure, digital dispensing, human reconstructed airway tissue, modified risk tobacco product screening, MucilAir, tobacco extract

\section{Introduction}

Inhalation hazards

T ODAY, THE SCOPE and number of potential lung toxicants have never been greater. Many of these materials of concern can be found in common products, including environmental materials, household products, personal care products, industrial chemicals, and pharmaceuticals. One area requiring further scrutiny is products for personal consumption such as tobacco products, with an increased focus on the expanding field of next-generation tobacco products that include electronic cigarettes (e-cig). While inhalation is the route of exposure for many new tobacco products, studies in animals are hampered by various deficiencies, including cost, lack of physiologically relevant exposures, and difficulty correlating the results to human data. These shortcomings and new regulatory demands have led to the development of many competent in vitrolex vivo models of lung toxicity. ${ }^{1}$

\section{Pulmonary models and respiratory toxicity}

Current nonanimal models include conventional twodimensional (2D) cell lines and primary cell cultures, as well as complex three-dimensional (3D) multicellular models, such as spheroids/organoids, precision-cut lung slices, and reconstructed human airway (RHuA) tissues. ${ }^{2-7}$ While

\footnotetext{
${ }^{1}$ Respiratory Toxicology Program, Institute for In Vitro Sciences, Inc., Gaithersburg, Maryland.

${ }^{2}$ Epithelix Sàrl, Geneva, Switzerland.

(c) Holger P. Behrsing et al., 2017; Published by Mary Ann Liebert, Inc. This Open Access article is distributed under the terms of the Creative Commons Attribution Noncommercial License (http://creativecommons.org/licenses/by-nc/4.0/), which permits any noncommercial use, distribution, and reproduction in any medium, provided the original author(s) and the source are credited.
} 
less complex 2D models have many merits, including costeffectiveness for screening events such as cytotoxicity, viability, or gene activation, they cannot model complex events that require multiple cell types. For example, inhalation of toxicants can result in harmful events in both the upper airways and the deep lung, depending on the nature of the insult.

Upper airway effects include irritation, sensitization, goblet cell hyperplasia, mucus secretion, and impaired cilia function. Multicellular 3D pulmonary models can be used to evaluate these complex events, specifically the RHuA model (derived from cells of bronchial origin) presents researchers with a more physiological platform that offers apical and basal compartments that allow flexibility in modeling a range of pulmonary exposures, including airway-like exposures at the apical surface (Fig. 1).

\section{In vitro/ex vivo exposures}

These 3D models can be used in conjunction with commercially available instruments that produce smoke and aerosols of tobacco products. This allows for the exposure of 3D RHuA tissues at the apical/airway site, which is the air-liquid interface (ALI) where gas exchange occurs. The use of these RHuA models can include omics and systems biology endpoints as part of a multifaceted approach to better understand the effects of tobacco product exposures. ${ }^{8-10}$ However, quantifying the materials deposited at the exposure site remains a challenge and prevents an accurate dose-response determination to be made. This very important issue has been the focus of interest at workshops (i.e., In Vitro Exposure Systems and Dosimetry Assessment Tools for Inhaled Tobacco Products, April 4-6, 2016, Bethesda, $\mathrm{MD}^{11}$ ) and remains a barrier to proper assessment of product liability.
Conventionally, this issue has been addressed by delivering a small volume of material-containing buffer to the apical site of an RHuA. While this allows a precise amount of material to be administered, perturbations can occur, including a disturbance in the mucous layer rheology, which inhibits the ability to quantify ciliary beat frequency (CBF) accurately. We are attempting to address this problem through a digital dispenser that allows picoliter droplet size dispensing. Using a coordinate system, the dispenser delivers a pattern of the desired test compound onto the apical surface of the tissue.

In this report, we evaluate a current RHuA model of bronchial airway epithelium (Epithelix MucilAir ${ }^{\mathrm{TM}}$ ). Following characterization of dispensing performance, we used an HP D300 digital dispenser to deliver precise amounts of vehicle (dimethyl sulfoxide; DMSO) or vehicle-containing tobacco extract (total particulate matter; TPM) to the apical side of RHuA tissues. The RHuA response was evaluated through cytotoxic (leakage marker), viability (WST-8 conversion), and functional (CBF) assays.

\section{Materials and Methods}

\section{Materials}

Digital dispensing was conducted using the HP D300 digital dispenser (Tecan, Morrisville, NC) and T8 dispense cartridges. Multiwell plates (96- and 24-well) were purchased from BD Falcon (Cat. nos. 353072 and 353047, respectively) and were used for coordinate dispense accuracy (96-well) tests and culture of Epithelix MucilAir tissues.

DMSO (Cat. no. 34869) for titration studies was obtained from Sigma-Aldrich (St. Louis, MO). Fluorescein disodium salt hydrate (Cat. no. A11659) was obtained from Alfa Aesar (Ward Hill, MA). Dulbecco's modified Eagle's medium

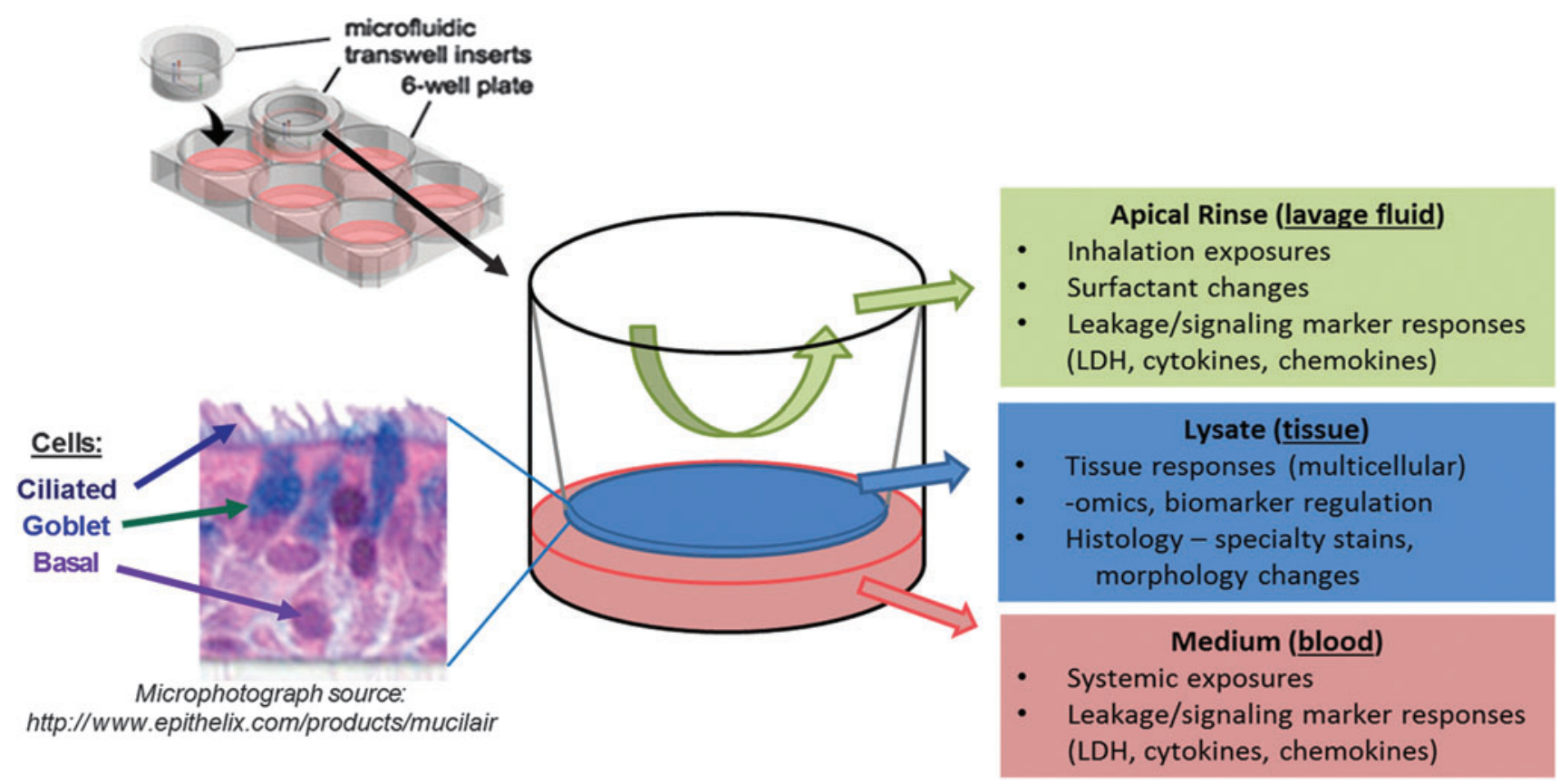

FIG. 1. A depiction of RHuA inserts and how they provide compartments is not available in other models. The micrograph insert shows multiple cell types, including basal, goblet, and ciliated epithelial cells, which can provide an enriched analysis not possible using cultures of single cell types. RHuA, reconstructed human airway. 
(DMEM) (Cat. no. 112-013-101) was purchased from Quality Biologicals, Inc. (Gaithersburg, MD).

TPM was obtained as a kind gift from Drs. Gaca and Haswell of British American Tobacco (BAT) and also from Dr. Tarran of the University of North Carolina (UNC). Preparations of TPM were created using ISO 3308: 2000 conditions (puffs consisting of $35 \mathrm{~mL}$ over 2 seconds, every 60 seconds) on an RM20 smoking machine (BAT) (Borgwaldt KC, Hamburg, Germany) or by using a Borgwaldt LC 1 Single Port engine (UNC) using the same puff volume and duration, but initiating puffs every 30 seconds.

MucilAir ${ }^{\mathrm{TM}}$ RHuA tissues were received from Epithelix Sàrl (Geneva, Switzerland) and consisted of tissues from one nonsmoking healthy male (28 years old; Batch no. 050401) and one nonsmoking female (48 years old; Batch no. 006501) who were Caucasian donors. RHuA tissues corresponding to the male donor were utilized on three separate occasions (run \#1, \#2, and \#3, referred to as M\#1, M\#2, and M\#3 henceforth) from three different seedings. Tissues (in one single seeding) from the female (F\#1) were run in parallel with M\#3. RHuA tissues were rinsed using MucilAir Culture Medium (MCM) 16-24 hours before any apical treatments. Medium was changed every 2-4 days, with visual inspection at every feeding or before treatment to visually confirm the health of cultures with beating cilia.

\section{Coordinate-based dispense accuracy protocol development}

Preliminary work evaluating the dispense patterns consisted of establishing plate layouts (e.g., assessing different plate formats such as 24-well plates vs. 96-well plates) and coordinate-based dispense patterns using Bio Deposition software by HP, Inc. (Palo Alto, CA). Visual evaluation and confirmation of dispense accuracy were conducted for both 96- and 24-well formats by placing a plate seal onto the lid, rim, or base of the wells or plate.

Dispensing of dye-spiked DMSO ( $\sim 15 \%$ red food color in DMSO or $5 \mathrm{mg} / \mathrm{mL}$ fluorescein-containing DMSO) was performed onto the plate seal and coordinate adjustments were made in the Bio Deposition software (if necessary) to ensure proper dispensing of patterns onto the desired location. Confirmation of proper dispensing was conducting by dispensing directly into wells and by confirming that dye staining was constrained to the location desired using visual observation. A dissecting or Nikon TE2000 laboratory-grade microscope was used to ensure dispense accuracy and that no streaking of dye was present along the lengths of the well wall, using light and/or fluorescence microscopy.

\section{Volume dispense accuracy}

The accuracy of D300 dispensing volumes in a patterned format was evaluated by using single-channel hand pipetting as a reference. Fluorescein stock solution $(5 \mathrm{mg} / \mathrm{mL}$ in DMSO) was diluted to $1 \mathrm{mg} / \mathrm{mL}$ in DMSO (F-DMSO) or TPM (F-TPM) solution from both BAT and UNC sources. Black-walled wells in 96-well plates (Cat. No. 6005225) from Perkin Elmer (Waltham, MA) receiving D300 pattern dispensing contained $100 \mu \mathrm{L}$ Hanks' buffered salt solution (HBSS), while those receiving hand pipetting contained $80 \mu \mathrm{L} \mathrm{HBSS}$.

For hand pipetting, F-DMSO or F-TPM was added to HBSS for a final concentration of $5000 \mathrm{~nL} / 100 \mu \mathrm{L}$ in
HBSS. This concentration of fluorescein-containing solution was serially diluted eight times using twofold dilutions. Four replicate wells per concentration of F-DMSO or F-TPM each received $20 \mu \mathrm{L}$ of the serially diluted solutions (and one set of nonspiked HBSS) so that a total volume range (per well) of $0.977-1000 \mathrm{~nL}$ of F-DMSO or F-TPM was created.

For digital dispensing, three different round patterns were generated, containing 52 (pattern 0.5D5.5-52), 69 (pattern 0.5D4.5-69), or 88 (0.5D5-88) spots per pattern, with spots $0.5 \mathrm{~mm}$ apart, arranged in a grid pattern that was confined within the well diameter of the plate. Patterns are coded based on the parameters used to create them, for example, $0.5 \mathrm{D} 5-88=$ droplet spacing is $0.5 \mathrm{~mm}$ apart, in a diameter of $5 \mathrm{~mm}$, using 88 spots. Droplet volumes ranged from 13 to $10,240 \mathrm{pL}$ per spot. Following dispensing and/or hand pipetting, plates were top read with six readings/well on a Flexstation plate reader by Molecular Devices (Sunnyvale, CA) using $485 \mathrm{~nm}$ (excitation) and $538 \mathrm{~nm}$ (emission) wavelengths, with a $530 \mathrm{~nm}$ cutoff.

\section{DMSO titration onto RHuA inserts}

Using a 24-well plate with the coordinates that successfully delivered the three patterns, a pattern of red dye inclusive DMSO ( 2 or $3 \mathrm{~nL} / \mathrm{spot}$ ) was delivered into empty inserts used by Epithelix for MucilAir cultures. Similar to the analysis of the coordinate dispense accuracy of 96-well plates, visual inspection and low-power microscopy of the inserts were used to determine whether delivery was complete and isolated to just the apical surface of the insert membranes. When necessary, the coordinates of delivery were adjusted so that delivery was accurate for inserts located at different locations of the plate. Dispensing onto live tissue was also tested for accuracy (using pattern 0.5D4.5-69 and $320 \mathrm{pL}$ droplet volume) and documented using F-DMSO. Within 1 minute of dispensing onto the apical surface of live tissue, fluorescein contained on the inserts was visualized and respective images digitized (Lumenera Infinity 2-1 camera) using the FITC channel.

\section{RHuA tissue exposure to DMSO titrations}

The same exposure conditions and DMSO dispense volumes used in the Volume dispense accuracy section were used to expose RHuA tissues. M\#1 and F\#1 each received a single dispense of material, while $\mathrm{M \# 2}$ received three dispenses (daily for 3 days) and were assayed for adenylate kinase $(\mathrm{AK})$ and viability on day 3 .

\section{Cytotoxicity of RHuA following titration}

AK cytotoxicity assays were conducted using the ToxiLight $^{\mathrm{TM}}$ Nondestructive Cytotoxicity BioAssay Kit (Cat. No. LT07-217) from Lonza (Walkersville, MD). Following the 72-hour treatment period, samples were collected for cytotoxicity evaluation. Apical rinses were conducted using $200 \mu \mathrm{L} \mathrm{HBSS}$. Lysis of RHuA was conducted in $0.5 \%$ Triton $\mathrm{X}-100$ in HBSS $(100 \mu \mathrm{L}$ apical, $400 \mu \mathrm{L}$ basal) for 2 hours at incubator conditions, 1 hour at incubator conditions with $3 \times$ trituration, or $16-24$ hours at $2^{\circ} \mathrm{C}-8^{\circ} \mathrm{C}$. Following the lysis period, the apical lysis and basolateral lysis volumes were pooled into one sample representing the tissue lysis. All samples in a comparison group (treatment vs. control) were handled in the same manner (e.g., lysis conditions), 
and all samples were stored at $\leq 60^{\circ} \mathrm{C}$ until the $\mathrm{AK}$ assay was run. The manufacturer's protocol for $\mathrm{AK}$ measurement was followed to obtain luminescence readings. In brief, $20 \mu \mathrm{L}$ samples were plated into a white-walled microplate (Perkin Elmer B\&W Isoplate; Cat. no. 6005060) incubated with $100 \mu \mathrm{L}$ reconstituted $\mathrm{AK}$ detection reagent, incubated for 5 minutes at RT, and read on a Berthold Orion luminometer microplate reader using 1 -second integrated read time.

\section{Viability of RHuA tissues following titration}

WST-8 viability assays were conducted using the CCK-8 kit (Cat. no. CK04-05) by Dojindo Molecular Technologies, Inc. (Rockville, MD). The active substrate solution (added to unmodified DMEM at a ratio of 1:10) was added to the apical $(150 \mu \mathrm{L})$ and basolateral $(350 \mu \mathrm{L})$ sides of the RHuA inserts in 24-well tissue culture plates (Cat. no. 353047; BD Falcon). Tissues were incubated for $150 \pm 30$ minutes before WST-8 absorbance measurements. Apical samples $(70 \mu \mathrm{L})$ or basolateral samples $(100 \mu \mathrm{L})$ were read for absorbance at $450 \mathrm{~nm}$ on a Molecular Devices Versamax plate reader. Samples from each absorbance value were compared with corresponding negative controls for apical and basolateral compartments, respectively, to determine whether loss of viability occurred.

\section{TPM digital dispensing versus hand pipetting}

For D300 dispensing, TPM was delivered using pattern 0.5D4.5-69 with four dispense volumes $(0,5.5,88.3$, and $706.6 \mathrm{~nL}$ total per insert). Matching DMSO volumes (except 0 , the same 0 untreated control insert's data were shared across the TPM and DMSO control datasets) were dispensed onto additional inserts to control for any DMSO effects. DMEM solutions created for hand pipetting were made using the same TPM stock and with matching amounts of TPM in each of the three concentrations tested in a $20 \mu \mathrm{L}$ sample. One $20 \mu \mathrm{L}$ aliquot was delivered through singlechannel pipette to the apical surface of each insert receiving its designated treatment. Figures derived from D300 TPM versus hand pipette dispensing onto inserts present $\mathrm{X}$-axis values based on the method or relevant unit for comparative/ informational purposes. Hand-pipetted samples indicate the TPM ( $\mu$ g/insert) contained in the respective $20 \mu \mathrm{L}$ aliquots delivered. The DMSO control group indicates the total DMSO dispense volume (nL/insert) and the D300 TPM dispense group lists the TPM exposure as $\mu \mathrm{g} / \mathrm{cm}^{2}$.

\section{Ciliary beat frequency}

CBF measurements were conducted using the SAVA system (Ammons Engineering, Clio, MI). Plates containing $\mathrm{RHuA}$ inserts were removed from the incubator and allowed to equilibrate to room temperature (15-20 minutes) before $\mathrm{CBF}$ analysis. Areas (avoiding the periphery of the insert) were videographed using $6 \times$ objective strength bright-field microscopy. Mucous balls/plugs occluding CBF measurements were avoided to obtain CBF fields that are usable. Average field motile points of less than 1000 were considered not evaluable (NE).

\section{Data analysis}

Titration-based datasets comprising three repeat trials with four replicate wells in each trial were averaged, and standard deviation (SD) was calculated. The \% CV as it pertains to each set of four replicate wells in a single trial was calculated by dividing the SD by the respective mean value and multiplying by 100 to obtain a $\% \mathrm{CV}$ of the mean for that dispense dataset. To generate intertrial \%CV values, the mean fluorescence intensity (MFI) and SD of each DMSO volume (hand pipetted or dispensed) from each trial were used to calculate the $\% \mathrm{CV}$ as was done for the individual trials.

Grubb's test was used to identify outlier wells whose data points were removed from the respective set of quadruplicate values for that group.

For cytotoxicity (\% AK release assay), percent released into the basolateral compartment or onto the apical surface was calculated. After plate background subtraction, apical or basolateral luminescence values were divided by total luminescence (apical+lysis + basolateral) to determine the percent of total released in each compartment. Alternatively, $\%$ of total may have been graphed using the $100 \%$ stacked column figure option in the Excel spreadsheet application (Microsoft Office Professional Plus 2010).

For viability calculations, the absorbance reading of treatment groups was calculated as a percentage of the untreated control to obtain $\%$ viability.

\section{Results}

\section{Coordinate-based dispensing}

Initial evaluation of pattern dispensing was conducted using red food color dye inclusion ( $\sim 15 \%$ in DMSO) whereby large droplets ( 2 or $3 \mathrm{~nL}$ each) were dispensed so that patterns could easily be recognized with the naked eye. Various patterns were evaluated by dispensing onto plate lids containing a plate seal tape (to prevent droplet movement via small electrostatic charges, etc.). It was subjectively determined that patterns are dispensed reproducibly and in the pattern established by the user in the HP Bio Pattern software (Fig. 2A, B). Droplets also appeared consistent, with the same shape and size, accurately placed in the target coordinates.

\section{Pattern dispensing accuracy}

To determine dispensing accuracy, three different round patterns were created, containing 52 (pattern 0.5D5.5-52), 69 (pattern 0.5D4.5-69), or 88 (0.5D5-88) spots per pattern, with spots $0.5 \mathrm{~mm}$ apart, arranged in a grid format (Fig. 3AC). The pattern dispense accuracy into wells of 96-well plates was tested by using the fluorescein tracer spiked into the DMSO being dispensed. Total well/pattern dispense volumes spanned from 0 to $901 \mathrm{~nL}$. Hand pipetting of serially diluted F-DMSO was conducted as a control, whereby the range of 0-1000 $\mathrm{nL}$ overlapped with all digitally dispensed pattern volumes. A comparison of MFI values demonstrated a very high level of consistency across replicate plates for hand pipetting and every pattern tested (Fig. 4A; Table 1).

The averaged MFI (across all repeat trials) had high concordance with that of the hand pipetting. However, the \%CV for replicate wells of patterns spanned a greater range. Hand pipetting yielded lower \%CV within the four replicate wells on a plate, but was higher when examining average MFI (for all DMSO concentrations) across repeat trials $(4.7 \% \mathrm{CV})$, as opposed to digital dispensing $(1.9 \%, 3.6 \%$, and $3.0 \%$ for 0.5D4.5-69, 0.5D5-88, and 0.5D5.5-52, respectively, for all 

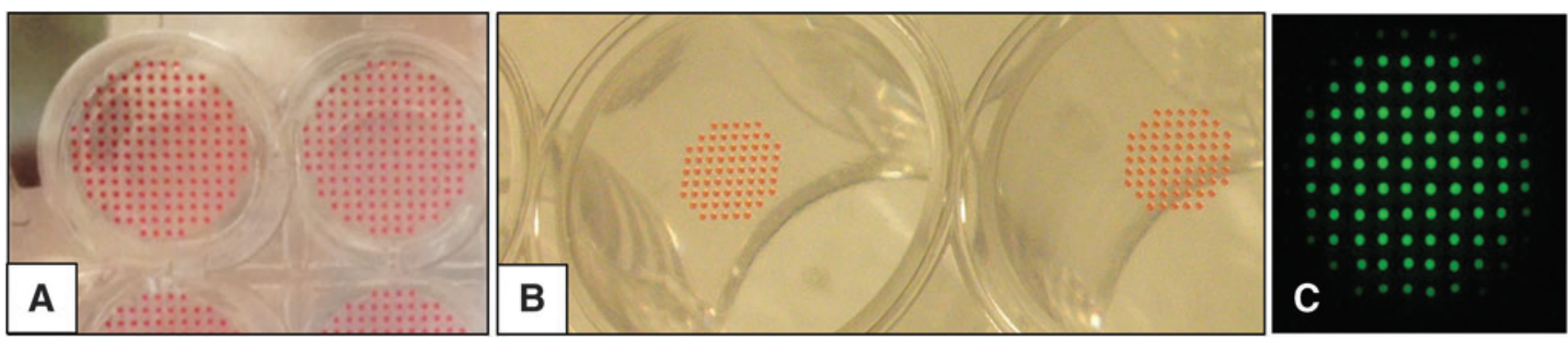

FIG. 2. Reproducible pattern deposition onto the plate lid of a 96-well plate (A) or onto center coordinates of a 24-well plate. (B) Red dye-containing DMSO droplets $(3 \mathrm{~nL})$ were in a $0.5 \times 0.5 \mathrm{~mm}$ grid onto their respective targets. (C) A fluorescein dye in DMSO was also used to verify patterning. DMSO, dimethyl sulfoxide.

volumes dispensed). In addition, of note was that one well's data were removed (using Grubb's test that showed it as an outlier) due to an apparent pipetting error. D300 dispensing was found to have higher average intraplate \% CV values. No correlation between droplet dispense volume and \%CV value was apparent, with the exception of the $450,560 \mathrm{pL}$ dispense volume for the 0.5D5-88 pattern where the \% CV for all three trials was higher $(\sim 19 \%)$. However, repeat trial reproducibility was more consistent using the D300 (as depicted by repeat trial dataset overlap in Fig. 4A and as calculated as intertrial \%CV in Table 1). Repeat trial average datasets also indicated that digital dispensing (for all three patterns) provides a greater concentration-dependent linearity $\left(\mathrm{R}^{2}\right.$ value; data not shown) of MFI.

\section{TPM dispensing accuracy}

Pattern 0.5D4.5-69 was utilized to test the D300 dispense capability for TPM material in DMSO. Two different sources of TPM (BAT and UNC) were spiked with fluorescein (using the same fluorescein spike volume as done for DMSO titration) and dispensed in three repeat trials using four replicate wells per trial. Hand pipetting of $20 \mu \mathrm{L}$ aliquots containing the same F-TPM volumes was used as a control. Hand pipetting demonstrated similar \%CVs as previously calculated for
DMSO titration. Dispensing of F-TPM (BAT) also had similar reproducibility as previously shown for the pattern used (data not shown). However, F-TPM (UNC) dispensing yielded two \%CV values above 20\% (22.3 and $24.1 \% \mathrm{CV}$ ) and $4 \% \mathrm{CV}$ values above $20 \%$ (three of them above $30 \%$ ) in the third trial (Table 2).

Comparing the linearity of titration, the MFI values of D300 F-TPM (BAT and UNC) were similar to that of handpipetting values, although the latter method yielded higher values at the two highest F-TPM volumes pipetted. Superimposing the averaged repeat trial F-DMSO data (hand pipette and 0.5D4.5-69 pattern) onto the F-TPM data, the MFI values produced a very similar slope (Fig. 4B). It should again be noted that one hand-pipette well value was found to be an outlier in its group and was removed from the quadruplicate well dataset.

The pattern of choice (0.5D4.5-69) was dispensed into the apical compartment by transferring the pattern to a 24 -well plate format. Red dye-spiked DMSO was used to confirm accurate dispensing (Fig. 5B). To evaluate the diffusion effect of dispensed material onto the mucous layer contained on the airway tissue, F-DMSO was dispensed into an insert containing live tissue. Visual observation using the FITC channel and time-lapse photography confirmed accurate pattern dispensing and depicted rapid diffusion in the insert (Fig. 5B) that

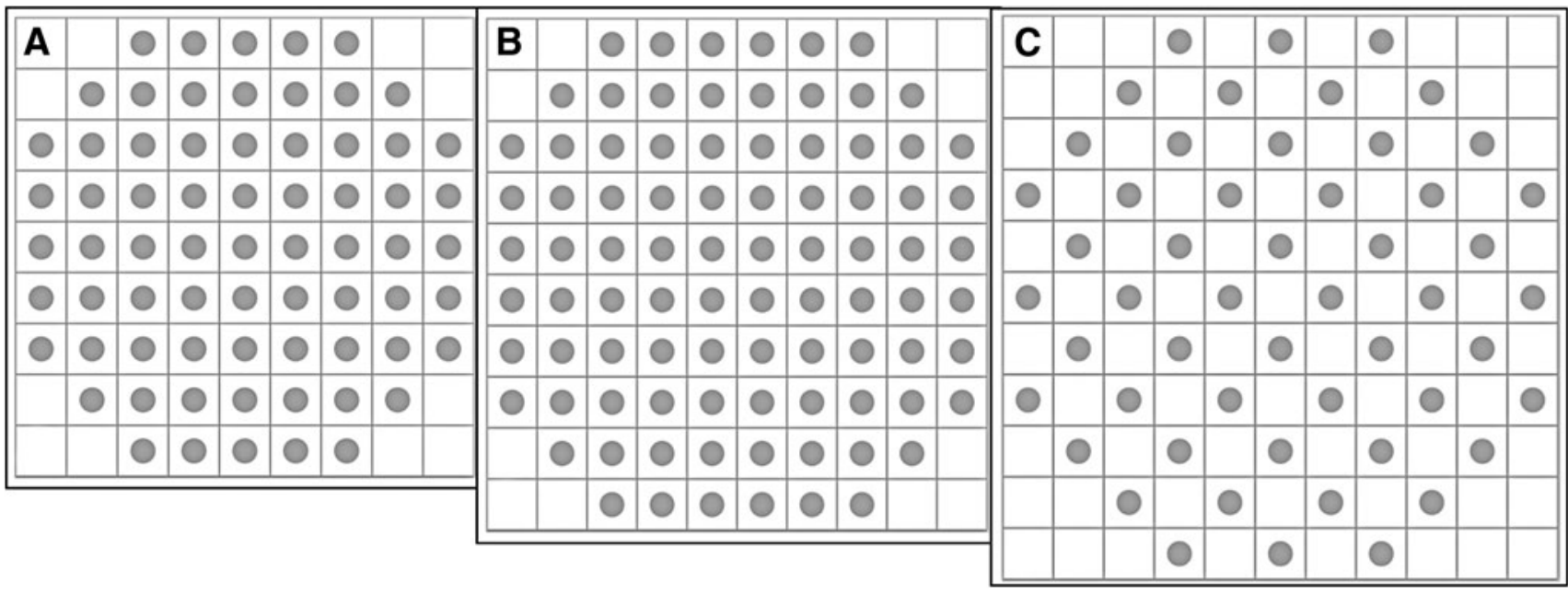

FIG. 3. Three patterns were created using an application-based coordinate system that allows specific spot placement. Each square represents a potential target coordinate that can be selected to create a pattern. (A) 0.5D4.5-69: a 69-spot pattern of 4.5 mm in diameter, (B) 0.5D5-88: an 88-spot pattern of 5.0 mm in diameter, and (C) 0.5D5.5-52: a 52-spot pattern of $5.5 \mathrm{~mm}$ in diameter. 

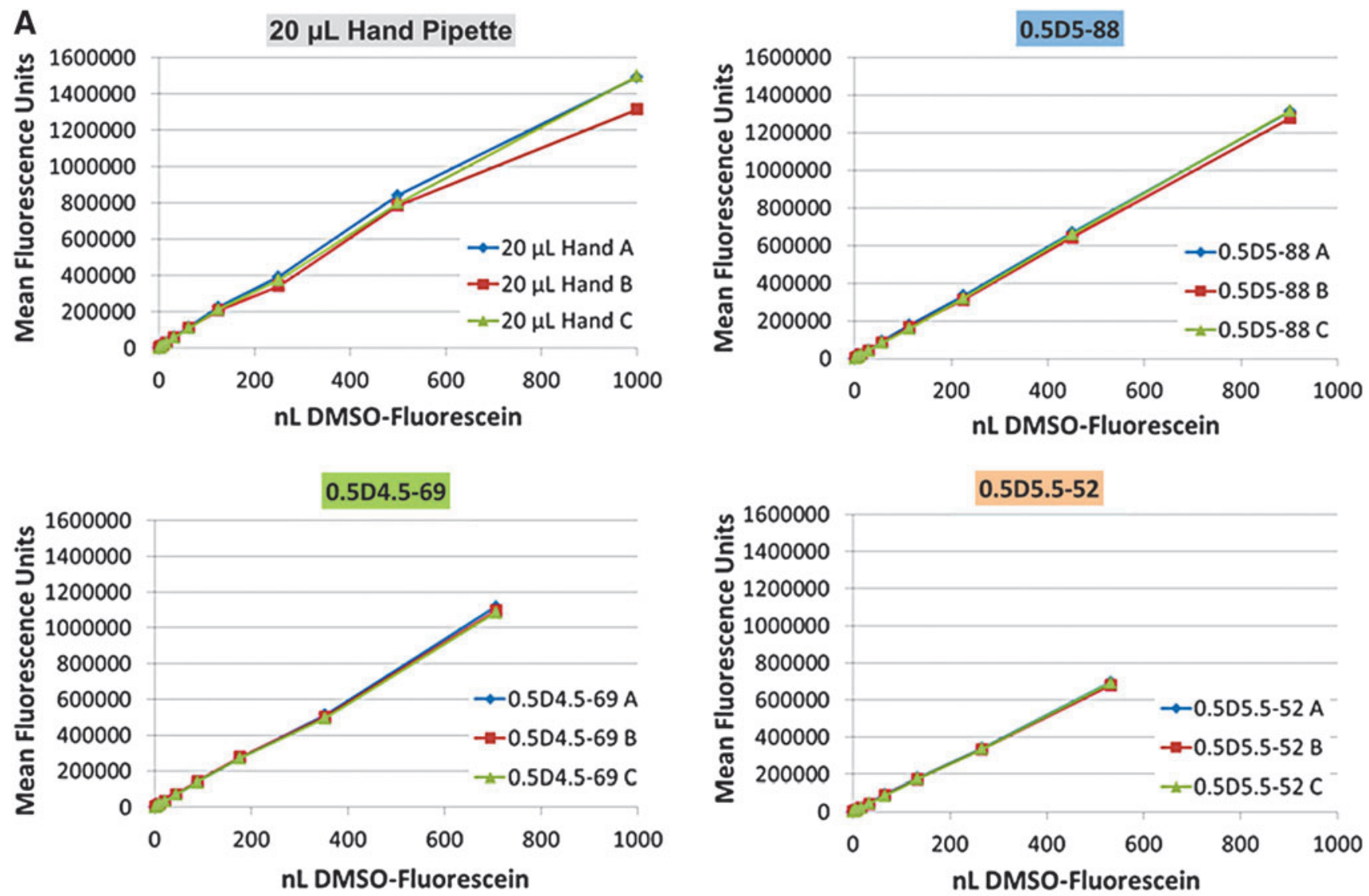

B

Titration Linearity

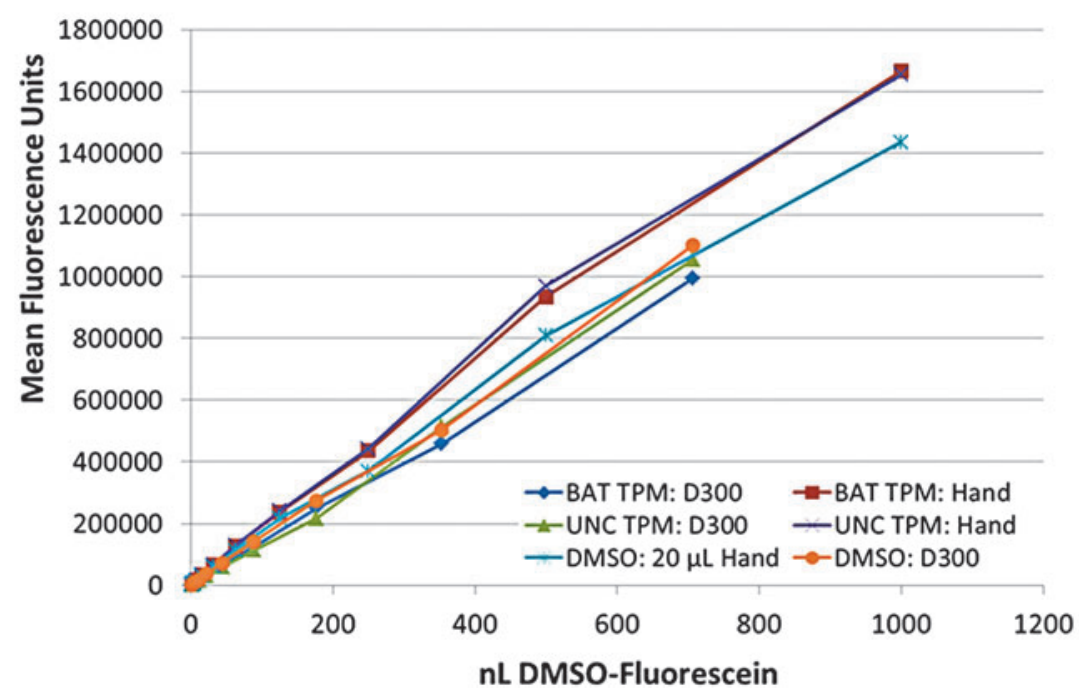

FIG. 4. (A) The mean fluorescence intensity of each pattern, along a titration gradient unique to each pattern due to droplet number, is shown. Hand pipetting (single channel, using $20 \mu \mathrm{L}$ aliquots to mimic an apical RHuA exposure) of serially diluted F-DMSO is used as a control to evaluate the reproducibility of each method of delivering a precise amount of DMSObased material into a microwell. Each pattern or hand pipetting was conducted three times (denoted as " $\mathrm{A}$," " $\mathrm{B}$," or " $\mathrm{C}$ " in the legend), using four replicate wells (averaged and represented by each data point) per attempt. (B) Dispensing F-TPM (BAT TPM and UNC TPM) is shown compared with hand pipetting the same F-TPM volumes in $20 \mu \mathrm{L}$ aliquots. Repeat trial averages of F-DMSO controls are included for comparison. 
TABle 1. DMSO Dispense Reproducibility

\begin{tabular}{|c|c|c|c|c|c|c|c|c|c|c|c|c|c|}
\hline \multirow{6}{*}{$\begin{array}{l}20 \mu \mathrm{L} \\
\text { Hand } \\
\text { pipette }\end{array}$} & Target $\mathrm{nL}$ & 1.0 & 2.0 & 3.9 & 7.8 & 15.6 & 31.3 & 62.5 & 125.0 & 250.0 & 500.0 & 1000.0 & Ave \\
\hline & Trial A & 1.4 & 1.4 & 1.0 & 0.7 & 0.7 & 1.6 & 0.9 & 0.9 & 0.8 & 6.1 & 0.7 & 1.5 \\
\hline & Trial B & 1.9 & 1.3 & 2.9 & 0.9 & 1.6 & 1.2 & 1.2 & 0.9 & 2.0 & 0.6 & 0.2 & 1.3 \\
\hline & Trial C & 2.8 & 1.7 & 2.6 & 0.9 & 1.1 & 0.4 & 0.8 & 0.2 & $1.5^{\mathrm{a}}$ & 1.0 & 0.5 & 1.2 \\
\hline & Ave $\% \mathrm{CV}$ & 2.0 & 1.5 & 2.2 & 0.8 & 1.1 & 1.1 & 1.0 & 0.7 & 1.4 & 2.6 & 0.5 & 1.3 \\
\hline & Intertrial \% CV & 7.0 & 4.3 & 7.1 & 3.0 & 2.7 & 3.5 & 2.4 & 4.1 & 6.9 & 3.5 & 7.3 & 4.7 \\
\hline \multirow[t]{6}{*}{$0.5 \mathrm{D} 4.5-69$} & $\mathrm{~nL} /$ pattern & 0.9 & 1.4 & 2.8 & 5.5 & 11.0 & 22.1 & 44.2 & 88.3 & 176.6 & 353.3 & 706.6 & Ave \\
\hline & Trial A & 7.9 & 4.2 & 7.8 & 11.3 & 14.2 & 10.4 & 6.0 & 4.7 & 3.4 & 4.9 & 4.7 & 7.2 \\
\hline & Trial B & 4.5 & 0.4 & 7.0 & 9.7 & 11.7 & 5.9 & 2.0 & 3.2 & 2.2 & 5.6 & 4.9 & 5.2 \\
\hline & Trial C & 4.7 & 0.9 & 8.0 & 9.4 & 9.2 & 6.2 & 2.0 & 1.9 & 2.7 & 3.8 & 5.8 & 5.0 \\
\hline & Ave $\% \mathrm{CV}$ & 5.7 & 1.9 & 7.6 & 10.1 & 11.7 & 7.5 & 3.3 & 3.2 & 2.8 & 4.8 & 5.1 & 5.8 \\
\hline & Intertrial \%CV & 3.8 & 1.8 & 1.0 & 1.3 & 2.8 & 2.4 & 1.9 & 1.0 & 1.5 & 1.8 & 1.5 & 1.9 \\
\hline \multirow[t]{6}{*}{$0.5 \mathrm{D} 5-88$} & $\mathrm{~nL} /$ pattern & 1.1 & 1.8 & 3.5 & 7.0 & 14.1 & 28.2 & 56.3 & 112.6 & 225.3 & 450.6 & 901.1 & Ave \\
\hline & Trial A & 4.7 & 1.8 & 7.1 & 9.0 & 7.9 & 6.5 & 4.3 & 4.3 & 3.7 & 18.7 & 5.5 & 6.7 \\
\hline & Trial B & 4.1 & 2.1 & 7.9 & 8.4 & 8.4 & 6.5 & 3.8 & 3.5 & 3.6 & 17.6 & 5.1 & 6.4 \\
\hline & Trial C & 4.9 & 1.9 & 7.6 & 9.1 & 8.3 & 7.3 & 4.5 & 4.3 & 3.8 & 19.4 & 6.1 & 7.0 \\
\hline & Ave $\% \mathrm{CV}$ & 4.6 & 1.9 & 7.5 & 8.8 & 8.2 & 6.7 & 4.2 & 4.0 & 3.7 & 18.6 & 5.5 & 6.7 \\
\hline & Intertrial \% CV & 2.6 & 6.8 & 3.8 & 1.5 & 2.8 & 4.0 & 5.0 & 5.9 & 3.3 & 2.1 & 1.6 & 3.6 \\
\hline \multirow[t]{6}{*}{$0.5 \mathrm{D} 5.5-52$} & $\mathrm{~nL} /$ pattern & 0.7 & 1.0 & 2.1 & 4.2 & 8.3 & 16.6 & 33.3 & 66.6 & 133.1 & 266.2 & 532.5 & Ave \\
\hline & Trial A & 2.0 & 0.2 & 2.0 & 2.9 & 2.9 & 2.0 & 2.6 & 2.8 & 2.3 & 0.7 & 11.5 & 2.9 \\
\hline & Trial B & 2.9 & 1.1 & 2.2 & 1.8 & 3.1 & 1.6 & 1.1 & 1.0 & 0.9 & 0.5 & 11.3 & 2.5 \\
\hline & Trial C & 0.6 & 2.7 & 0.7 & 2.5 & 2.8 & 0.9 & 2.0 & 1.7 & 1.2 & 0.9 & 11.0 & 2.4 \\
\hline & Ave $\% \mathrm{CV}$ & 1.8 & 1.3 & 1.6 & 2.4 & 2.9 & 1.5 & 1.9 & 1.8 & 1.5 & 0.7 & 11.3 & 2.6 \\
\hline & Intertrial \% CV & 2.5 & 5.2 & 4.2 & 2.5 & 2.6 & 3.6 & 3.9 & 4.2 & 1.8 & 0.8 & 1.1 & 3.0 \\
\hline
\end{tabular}

${ }^{a}$ Indicates one replicate data point was removed as an outlier.

Percent coefficient of variation (\%CV) for MFI values are shown for each method/pattern tested. Hand pipetting of $20 \mu \mathrm{L}$ aliquots of FDMSO containing HBSS is compared with a titration of F-DMSO droplet sizes delivered in three patterns containing different numbers of droplets that are pattern specific. Trial A-C: each trial consists of one set of four replicate wells and the \%CV values shown are calculated for each set of wells in a trial; Ave \% CV: an average \% CV calculated for all three replicate trials. Intertrial \% CV: the average \% CV for all three trials, calculated using each trial's Ave MFI and SD value for the respective dispense volume. The Ave values listed (right-most column) are an average $\mathrm{CV}$ value across all volumes dispensed.

HBSS, Hanks' buffered salt solution; MFI, mean fluorescence intensity; SD, standard deviation.

appeared to equilibrate within 10-20 minutes, depending on the insert. No overt differences were noted due to donor.

\section{Assessment of potential cytotoxicity or loss of viability}

To evaluate the potential cytotoxicity of titrated DMSO, RHuA tissues received a titration of DMSO volumes using the 0.5D4.5-69 pattern and apical rinse, basolateral medium and lysate samples were collected following 72 hours of postexposure and assayed for $\mathrm{AK}$ content in each sample type/ compartment. Results demonstrate that $\mathrm{AK}$ quantities were minimal in both basolateral and apical compartments for all concentrations through $177 \mathrm{~nL}$ total DMSO (Table 3). However, the $707 \mathrm{~nL}$ dispense volume elicited a greater AK release trend into the nontissue compartments, with tissue lysate quantities also diminished compared with the untreated control.

To assess viability, apical and basolateral samples collected after WST-8 assay incubation were plated and read for absorbance to determine the relative quantities of enzymatic-based substrate conversion as a function of

Table 2. Total Particulate Matter Dispense Reproducibility

\begin{tabular}{|c|c|c|c|c|c|c|c|c|c|c|c|c|c|}
\hline \multirow{6}{*}{$\begin{array}{l}20 \mu \mathrm{L} \\
\text { Hand } \\
\text { pipette }\end{array}$} & Target $\mathrm{nL}$ & 1.0 & 2.0 & 3.9 & 7.8 & 15.6 & 31.3 & 62.5 & 125.0 & 250.0 & 500.0 & 1000.0 & Ave \\
\hline & Trial A & 2.6 & 2.9 & 2.6 & 2.7 & 2.0 & 0.9 & 1.5 & 2.4 & 1.1 & 0.3 & 1.8 & 1.9 \\
\hline & Trial B & 2.2 & 1.4 & 2.9 & 3.5 & 3.9 & 4.8 & 4.3 & 4.5 & 1.6 & $1.7^{\mathrm{a}}$ & 1.0 & 2.9 \\
\hline & Trial C & 1.9 & 1.7 & 3.7 & 1.9 & 3.3 & 3.4 & 4.9 & 4.0 & 1.7 & 0.8 & 0.4 & 2.5 \\
\hline & Ave $\% \mathrm{CV}$ & 2.3 & 2.0 & 3.1 & 2.7 & 3.1 & 3.0 & 3.6 & 3.6 & 1.5 & 1.0 & 1.1 & 2.4 \\
\hline & Intertrial \%CV & 0.8 & 2.0 & 2.6 & 3.2 & 1.2 & 3.3 & 2.3 & 3.2 & 3.1 & 1.0 & 3.7 & 2.4 \\
\hline \multirow{6}{*}{$\begin{array}{l}0.5 \mathrm{D} 4.5-69 \\
\text { (UNC F- } \\
\text { TPM) }\end{array}$} & nL/pattern & 0.9 & 1.4 & 2.8 & 5.5 & 11.0 & 22.1 & 44.2 & 88.3 & 176.6 & 353.3 & 706.6 & Ave \\
\hline & Trial A & 6.7 & 2.5 & 6.6 & 12.2 & 18.4 & 15.5 & 16.0 & 17.4 & 11.0 & 18.1 & 7.7 & 12.0 \\
\hline & Trial B & 9.1 & 1.7 & 7.0 & 12.1 & 16.9 & 15.8 & 12.7 & 22.3 & 24.1 & 5.8 & 7.7 & 12.3 \\
\hline & Trial C & 9.1 & 8.0 & 17.4 & 18.6 & 33.7 & 37.3 & 31.1 & 22.7 & 15.4 & 6.9 & 5.4 & 18.7 \\
\hline & Ave $\% \mathrm{CV}$ & 8.3 & 4.1 & 10.3 & 14.3 & 23.0 & 22.8 & 19.9 & 20.8 & 16.8 & 10.3 & 6.9 & 14.3 \\
\hline & Intertrial \%CV & 6.5 & 7.7 & 17.5 & 28.2 & 31.9 & 34.7 & 36.9 & 40.1 & 39.8 & 13.5 & 12.8 & 24.5 \\
\hline
\end{tabular}

${ }^{a}$ Indicates one replicate data point was removed as an outlier.

Percent coefficient of variation (\%CV) for MFI values are shown for hand pipetting of $20 \mu \mathrm{L}$ aliquots of F-TPM (UNC) containing HBSS compared with a titration of F-TPM (UNC) titrated using pattern 0.5D4.5-69. Trial A-C: each trial consists of one set of four replicate wells and the \% CV values shown are calculated for each set of wells in a trial; Ave \% CV: an average \% CV calculated for all three replicate trials. Intertrial \% CV: the average \% CV for all three trials, calculated using each trial's Ave MFI and SD value for the respective dispense volume. The Ave values listed (right-most column) are an average CV value across all volumes dispensed.

TPM, total particulate matter. 

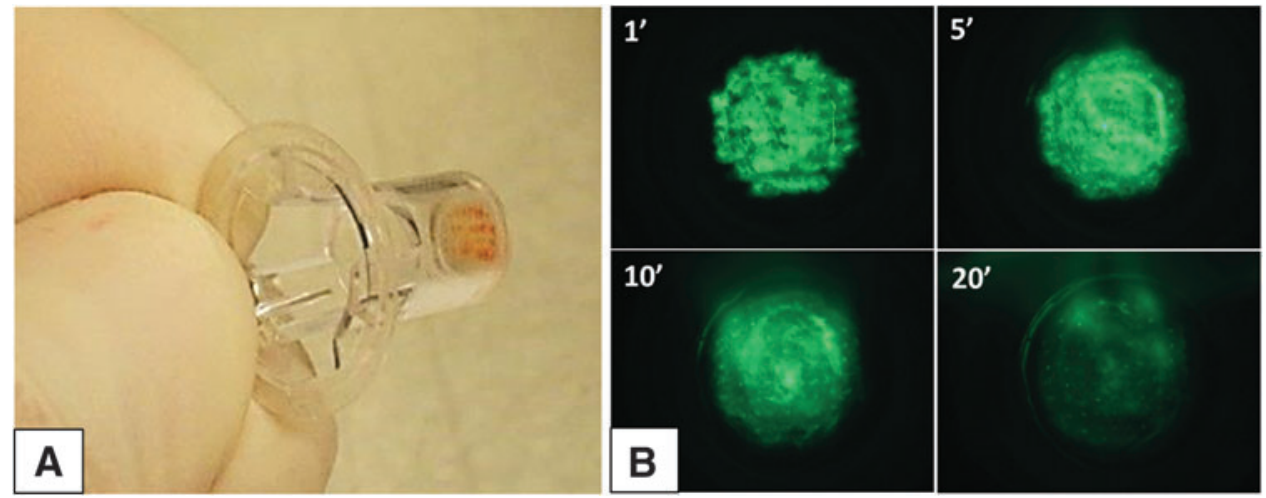

FIG. 5. (A) Red dye-spiked DMSO was dispensed using pattern 0.5D4.5-69 onto the apical membrane surface of an empty RHuA insert. (B) Digital dispensing of F-DMSO using pattern 0.5D4.5-69 showed accurate deposition and rapid diffusion of material on the apical surface of an RHuA. Images displayed represent $1,5,10$, and 20 minutes following dispensing.

viability. Results suggest that a single exposure (or hand pipetting) may not result in a loss of viability, but that repeat exposures can decrease viability in comparison with the untreated control group (Fig. 6).

\section{Ciliary beat frequency}

The SAVA system was used to measure CBF of pre- and postdispense, room temperature-acclimated RHuA tissues receiving hand-pipetted HBSS buffer (or MCM for TPM studies) or digitally dispensed DMSO or TPM. This pilot study evaluating $\mathrm{CBF}$ (for M\#1, M\#3, and F\#1 tissues) yielded varied baseline frequencies, appearing to be insert (and not treatment) specific. For example, before any treatment, M\#1, F\#1, and M\#3 exhibited CBF ranges of 3.5-7.7, 4.8-6.8, and 5.18.3 , respectively. Visual observation of hand-pipetted buffer onto the RHuA inserts resulted in an overt increase in mucous/particulate matter movement on the apical surface of the insert. The RHuA tissues receiving daily applications of $20 \mu \mathrm{L}$ hand pipetting were found to retain apical buffer volume during the course of the 3-day study. Digital dispensing did not result in any overt change in the rheology of the apical surface of inserts as viewed microscopically.

Of note was that the highest volume of DMSO digitally dispensed $(707 \mathrm{~nL})$ resulted in a cessation of ciliary beating,

Table 3. Percent of Total Adenylate Kinase

\begin{tabular}{|c|c|c|c|c|c|c|c|c|c|}
\hline \multirow{2}{*}{$\frac{\text { Donor }}{\text { Compartment }}$} & \multicolumn{3}{|c|}{ M\#1 } & \multicolumn{3}{|c|}{$F \# 1$} & \multicolumn{3}{|c|}{$M \# 2$} \\
\hline & $A R$ & $T L$ & $B M$ & $A R$ & $T L$ & $B M$ & $A R$ & $T L$ & $B M$ \\
\hline Volume & Han & d pipe & & & & & & & \\
\hline $20 \mu \mathrm{L}$ HBSS & 0.8 & 98.6 & 0.6 & 0.8 & 99.2 & 0.0 & 0.2 & 99.7 & 0.1 \\
\hline nL DMSO & D3C & $0(0.5$ & $4.5-6$ & & & & & & \\
\hline 0 & 0.7 & 98.7 & 0.5 & 0.5 & 99.5 & 0.0 & 0.0 & 99.9 & 0.1 \\
\hline 3 & 1.4 & 98.3 & 0.3 & 0.4 & 99.6 & 0.0 & 0.9 & 99.1 & 0.0 \\
\hline 6 & 2.2 & 95.2 & 2.5 & 1.0 & 99.0 & 0.0 & 0.5 & 99.5 & 0.0 \\
\hline 11 & 1.0 & 98.5 & 0.5 & 0.4 & 99.6 & 0.0 & 0.2 & 99.9 & 0.0 \\
\hline 22 & 0.6 & 99.1 & 0.3 & 0.3 & 99.7 & 0.1 & 0.2 & 99.8 & 0.0 \\
\hline 44 & 0.8 & 98.9 & 0.3 & 0.6 & 99.4 & 0.0 & 0.2 & 99.6 & 0.2 \\
\hline 88 & 2.0 & 97.3 & 0.8 & 1.5 & 98.4 & 0.1 & 0.6 & 99.3 & 0.1 \\
\hline 177 & 1.3 & 98.2 & 0.6 & 6.1 & 93.4 & 0.5 & 0.6 & 99.3 & 0.1 \\
\hline 707 & 6.3 & 87.0 & 6.6 & 9.1 & 81.2 & 9.7 & 11.4 & 86.8 & 1.8 \\
\hline
\end{tabular}

A compartment-based breakdown of AK percent of each RHuA insert is given. Apical rinse (AR), Tissue lysate (TL), and basolateral medium (BL) levels are compared. $\mathrm{M \# 1}$ and $\mathrm{F} \# 1$ received a single dispense and $\mathrm{M \# 2}$ received three daily dispenses. Samples were collected at day 3 for analysis.

RHuA, reconstructed human airway. although small areas containing beating cilia were observed at the periphery of the insert (where CBF measurements are typically avoided). However, the $\%$ active area output from SAVA analysis captures the exposure-induced loss of beating cilia that is prevalent in the majority of the insert surface area (Table 4). This potential marker of exposure-induced change will be explored further as a means to detect or quantify quasi-blockage.

It should be noted that although no loss of active area was visually observed with hand pipetting (i.e., actively beating cilia are still observed beneath the moving debris), a decrease in active area is calculated by SAVA within 24 hours of the volume delivery. However, it was found that 24 hours postdispense, the ciliary beat began to return for the insert receiving this DMSO volume, although the number of motile points remained lower than predispense values. Daily repeated dispensing (M\#3) resulted in the second highest DMSO volume (177 nL) also yielding a diminished active area of ciliary beating (data not shown) following the second dispense (conducted at 24 hours, postfirst dispense).

Titration of TPM onto RHuA tissues was conducted using three volumes of TPM (achieving three amounts of TPM on the airway surface) and a $0 \mathrm{~nL}$ DMSO control. To match TPM volumes digitally dispensed, one $20 \mu \mathrm{L}$ volume of unspiked MCM control was used as a 0 TPM control, while

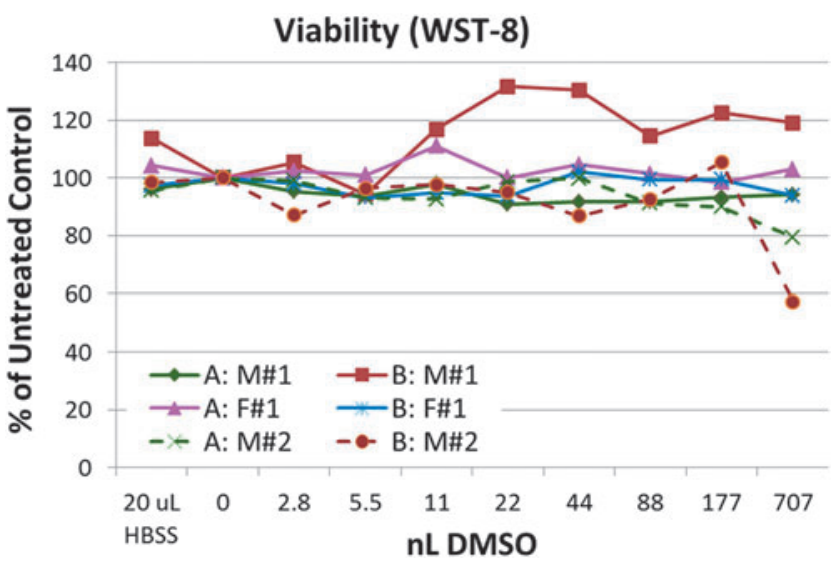

FIG. 6. Assessment of viability in apical (A) and basolateral (B) compartments. No significant loss of viability is detected using a single dispense. M\#2 receiving three dispenses demonstrated an apical \% viability of $79 \%$, while the basal compartment yielded less than $60 \%$ of untreated control (0 data point). 
Table 4. Active Area (\%) of Selected CBF Fields

\begin{tabular}{|c|c|c|c|c|c|c|c|c|}
\hline Donor & Treatment & $\begin{array}{l}0.25 \text { hour } \\
\quad(\text { Pre })\end{array}$ & $\begin{array}{l}0.25 \text { hour } \\
\quad(\text { Post })\end{array}$ & 24 hours & $\begin{array}{c}24 \text { hours } \\
(\text { Post })\end{array}$ & 48 hours & $\begin{array}{c}48 \text { hours } \\
\text { (Post) }\end{array}$ & 72 hours \\
\hline \multirow[t]{4}{*}{ F\#1 } & $\begin{array}{l}20 \mu \mathrm{L} \\
\text { HBSS }\end{array}$ & 54 & 3 & 18 & \multirow[t]{4}{*}{ NA } & 79 & \multirow[t]{4}{*}{ NA } & 73 \\
\hline & $0 \mathrm{~nL}$ & 71 & 60 & 69 & & 75 & & 84 \\
\hline & $44 \mathrm{~nL}$ & 76 & 43 & 68 & & 47 & & 84 \\
\hline & $707 \mathrm{~nL}$ & 69 & 1 & 9 & & 17 & & 26 \\
\hline \multirow[t]{4}{*}{ M\#3 } & $\begin{array}{l}20 \mu \mathrm{L} \\
\text { HBSS }\end{array}$ & 17 & 19 & 12 & 2 & 10 & 1 & 15 \\
\hline & $0 \mathrm{~nL}$ & 41 & 54 & 55 & 83 & 55 & 47 & 76 \\
\hline & $44 \mathrm{~nL}$ & 34 & 50 & 46 & 77 & 52 & 41 & 66 \\
\hline & $707 \mathrm{~nL}$ & 64 & 8 & 3 & 0 & 15 & 1 & 1 \\
\hline
\end{tabular}

NA, not applicable as repeat deliveries were not made to F\#1 inserts.

SAVA-calculated $\%$ active area is presented on a time point basis, either predispense (-) or at the hours indicated postdispense for handpipetted (HBSS) or digitally dispensed (DMSO) volumes. F\#1 received a single dispense and CBF was monitored for up to 72 hours. M\#3 received daily additions of hand pipette or D300 dispensing of the volumes (nL) shown, with a postdispense measurement (post) taken immediately following dispensing.

three additional $20 \mu \mathrm{L}$ volumes, each containing the same volumes of TPM digitally dispensed, were hand pipetted onto the apical surface of RHuA. Neither hand-pipetted nor D300-dispensed TPM appeared to cause a loss of viability (Table 5) or cause cytotoxicity (data not shown). As shown with DMSO titration studies, the highest volume of DMSO pipetted may have elicited a small increase in apical or basolateral medium ( $<10 \%$ where detected), but this was not consistently observed across the donor tissues or across seedings.

\section{Discussion}

\section{RHuA and dispensing}

Current, state-of-the-art RHuA cultures offer researchers a multicellular model by which more complex events or functionality of human airways can be modeled. Models available include those modeling the nasopharyngeal and bronchial region and, recently, also those modeling small airways and alveolar regions. The advancement and greater variety of these more physiologically relevant models will make them more applicable for studies involving potential pulmonary toxi- cants, such as e-cigs or other next-generation tobacco products. However, despite the availability of various machines for generating smoke and aerosols, ${ }^{3,12-15}$ analytical instruments to measure quantities and chemical constituents within them have not been established. ${ }^{16,17}$ In addition, a standardized paradigm has not been established to quantify materials at the exposure site; a subject of breakout group discussions at a recent workshop ("In Vitro Exposure Systems and Dosimetry Assessment Tools for Inhaled Tobacco Products," April 4-6, 2016, Bethesda $\mathrm{MD}^{11}$ ) that is the second of a series of tobacco-related workshops intended to address and promote the use of in vitro nonanimal methods to foster an ethical research paradigm for new tobacco product development.

We evaluated the HP D300 as a means to deliver a low volume of test material, to a specific location (i.e., apical/airway surface of RHuA), through direct dispensing onto the mucous layer. This characterization involved assessing the accuracy of dispense location and volume in a pattern-specific manner. The initial performance assessment in 96-well format (having the same well surface area as the MucilAir tissue culture insert membrane) allowed subsequent testing directly onto RHuA

Table 5. Reconstructed Human Airway Viability Following a Single Total Particulate Matter Pipette or Dispense onto Reconstructed Human Airway Tissues

\begin{tabular}{cccccccccc} 
& \multicolumn{3}{c}{ Hand pipette } & \multicolumn{3}{c}{ D300: DMSO control } & \multicolumn{3}{c}{ D300: TPM } \\
\cline { 2 - 11 } Donor & $\mu$ Total & $A$ & $B$ & $n L$ Total & $A$ & $B$ & $\mu g / \mathrm{cm}^{2}$ & $A$ & $B$ \\
\hline \multirow{3}{*}{ M\#2 } & 0.0 & 100.0 & 100.0 & 0.0 & 100.0 & 100.0 & 0.0 & 100.0 & 100.0 \\
& 0.1 & 102.8 & 100.4 & 5.5 & 108.8 & 121.7 & 0.4 & 107.8 & 95.1 \\
& 2.1 & 102.2 & 106.5 & 88.3 & 112.3 & 127.0 & 6.4 & 104.6 & 108.5 \\
& 17.0 & 98.5 & 110.8 & 706.6 & 111.9 & 110.7 & 51.1 & 103.9 & 83.9 \\
M\#3 & 0.0 & 100.0 & 100.0 & 0.0 & 100.0 & 100.0 & 0.0 & 100.0 & 100.0 \\
& 0.1 & 112.3 & 91.7 & 5.5 & 102.6 & 96.2 & 0.3 & 105.1 & 95.1 \\
& 1.4 & 106.4 & 82.1 & 88.3 & 97.5 & 96.2 & 4.3 & 101.8 & 90.8 \\
& 11.3 & 123.2 & 86.4 & 706.6 & 104.1 & 97.3 & 34.1 & 99.9 & 82.0 \\
F\#1 & 0.0 & 100.0 & 100.0 & 0.0 & 100.0 & 100.0 & 0.0 & 100.0 & 100.0 \\
& 0.1 & 107.3 & 112.4 & 5.5 & 102.2 & 101.0 & 0.3 & 104.5 & 111.0 \\
& 1.4 & 108.8 & 115.5 & 88.3 & 103.3 & 90.3 & 4.3 & 101.6 & 100.9 \\
& 11.3 & 107.6 & 103.7 & 706.6 & 96.6 & 88.0 & 34.1 & 118.4 & 102.6
\end{tabular}

Apical (A) and basolateral medium (B) viability readings are listed as $\%$ of the negative control (20 $\mu \mathrm{L}$ HBCC for hand pipetting or 0 DMSO for D300 dispensing). BAT TPM was administered to the M\#2 Donor, while M\#3 and F\#1 received UNC TPM. To control for the DMSO dispensed with TPM, a second series of tissues (D300: DMSO Control) received corresponding volumes of DMSO. Hand-pipetted TPM amounts corresponded to those delivered with the D300. 
tissues whereby the tolerance (via cytotoxicity and viability endpoints) and $\mathrm{CBF}$ response of tissues to this exposure method were examined.

\section{Coordinate accurate dispensing}

Qualitative evaluation of coordinate-based pattern dispensing concluded that the dispenser dispensed reliably and accurately. However, although the D300 is known to have a very low CV for single spot deliveries, understanding whether dispensing a pattern array of spots magnifies the error is very important. Using hand-pipetting serial dilutions of F-DMSO as the benchmark, multiple trails demonstrated excellent concordance of all three digitally dispensed patterns with respect to fluorescein signal. Although hand-pipetted CVs may have been lower within a trial, repeated trials demonstrated very consistent digital dispensing across these trials.

The D300 dispense cartridges are reportedly capable of dispensing suspensions and this was verified with F-TPM dispensing, although higher CVs were seen with some wells using TPM of the UNC source. With the D300 having dispensed into wells with low CVs after dispensing into wells with higher $\mathrm{CVs}$, this is difficult to explain in terms of particulates clogging the dispense channels unless unclogging occurred after high CV wells. It is also conceivable that particulate matter was trapped in the dispense channels, but soluble fluorescein in DMSO was able to dispense without the particulate matter. Not included in the hand-pipetted data (removed as outliers) is one well in each of the FDMSO and F-TPM trials where approximately double the fluorescein signal was measured. This was likely due to mispipetting and highlights the difficulty of hand pipetting large numbers of wells using a single-channel pipette without error; a nonissue for the $\mathrm{D} 300$.

\section{Dispense quantity accuracy}

The D300 was designed to deliver small amounts of material into multiwell plates and has demonstrated this accurately with patterns as well. However, the goal of the study conducted here was to use the D300 as a means to deliver an accurate pattern containing test material onto the apical surface of an insert containing RHuA tissues. Development of dispensing protocols included the selection of a pattern with a smaller dispense diameter than the insert membrane diameter found on the RHuA. This was required because although the culture inserts hang centered from the upper well point of contact, the inserts do allow a small amount of play where they may shift slightly as the D300 rapidly moves from well to well dispense coordinate. This also indicates that each RHuA insert may not receive a pattern directly in the center of the insert. Even if it does deliver the pattern centered, the periphery of the tissue will not receive dispensed droplets with a smaller diameter pattern. Furthermore, the coordinate system currently allows no smaller than a $0.5 \mathrm{~mm}$ spot-spot dispensing distance interval.

The two points raised indicate there will be dispense areas that will have higher concentrations of material than those areas where droplets do not deploy. However, the inherent properties of multicellular RHuA tissue may make this a moot point. Not only do RHuA tissues exhibit an apical mucous layer, ${ }^{18-20}$ but also fully mature MucilAir tissues yield an estimated 3-5 $\mu \mathrm{L}$ of mucous volume at equilibrium in ad- dition to beating cilia when fully mature. Collectively, the cilia-driven mucous layer was expected to quickly disperse materials dispensed with the D300 (Fig. 4B). Multiple FDMSO dispenses on two different tissues showed similar dye dispersion rates, although differences in staining intensity may have been dependent on donor/RHuA tissue-specific mucous volume and/or viscosity. While donor-specific differences may always play a role in test material dispersion (or adverse effects thereof), a standardized protocol for RHuA tissue rinse before test material exposure will minimize variables attributable to excessive mucous buildup or lack thereof if researchers rinse just before exposure-the latter a seemingly nonphysiologically relevant paradigm given that human lungs inherently have a mucous or surfactant layer along the entire airway continuum.

\section{RHuA tolerance}

Dispensing a range of DMSO volumes onto the RHuA was intended to establish where the DMSO volume breaking point may be. With a low microliter mucous volume, it was expected that low nanoliter DMSO/insert would result in marker leakage and/or reduced viability. However, only the highest DMSO volume dispensed (707 nL) elicited some AK release (but no loss of viability as measured using the WST-8 assay). Using a multidispense exposure paradigm, the daily DMSO dispensing for three consecutive days (conducted for M\#3) did indeed result in lower viability, although the AK assay did not reflect a greater basolateral AK level. For the rapid diffusion rates of DMSO combined with tissue volume and $700 \mu \mathrm{L}$ of medium playing into the final DMSO concentration, a number of dispenses should be possible without reading a $0.1 \%$ final concentration in the entire distribution volume available.

With TPM-exposed RHuA tissues (up to 34 or $51 \mu \mathrm{g}$ of TPM from UNC and BAT sources, respectively) not exhibiting a TPM-based loss of viability, repeat exposure, and expanded time course, studies are warranted that will also increase physiological relevance. One published report of a prolonged epithelial culture suggests that nonlethal changes can occur in in vitro models. ${ }^{21}$ The additional endpoints such future studies may elucidate could allow researchers to explore more subtle exposure-related effects. It is fully understood that optimizing the time course, including that for subcytotoxic loss of epithelial barrier function, cytokine release, or gene regulation, may elucidate other aspects of biopattern dispensing relevant to the focus of the study goals. Ongoing work with other markers of interest that may be induced by digital dispensing will continue to characterize dispensing effects on RHuA tissues. The initial pilot work reported here establishes some baseline parameters that this new exposure method affords scientists.

\section{Ciliary beating}

An inherent disadvantage to hand pipetting a buffersolubilized test material to the apical surface of an RHuA is the perturbation of apical surface rheology. Researchers evaluating $\mathrm{CBF}$ often abstain from measurements for a number of hours before readings. While RHuA tissues are known to effectively resorb excess fluid on the airway surface, ${ }^{20}$ this can require a number of hours or days, depending on the volume delivered and donor-specific qualities of the tissues. ${ }^{22}$ 
The work presented here evaluated hand-pipetted buffer exposure and digitally dispensed DMSO titrations to the airway surface of RHuA. As expected and noted visually, hand pipetting a $20 \mu \mathrm{L}$ volume to the apical surface caused a substantial disturbance of the apical rheology, with particulates swirling in a circular manner as the material is driven by beating cilia. SAVA assessment of CBF in these groups suggested that a loss of ciliary beating may occur as noted with a lower calculated \% active area, but visual observation suggests otherwise as ciliary beating is clearly observed beneath the swirling debris. This supports the general belief that accurate $\mathrm{CBF}$ measurements may not be possible with movement of large numbers of particles on the apical surface. The loss of active area (as interpreted by the SAVA analysis) may reflect a nonideal data source for analysis, but it should not be ruled out that this may, in part, be due to a light refraction change (due to increased apical volume) interfering with SAVA data analysis; an adjustment of microscope settings may help resolve it.

D300-dispensed DMSO did not appear to readily disturb the apical rheology of the RHuA tissues when dispensing up to $707 \mathrm{~nL}$ (no moving debris or mucous was noted). However, this volume did inhibit ciliary beating for both donors (M\#1 \& F\#1) receiving a single dispense. Repeated dispense (M\#3) elicited a ciliary beating loss at the next lower total volume dispensed (177 $\mathrm{nL})$. A dispense volume-dependent change in $\mathrm{CBF}$ was not noted, but the baseline $\mathrm{CBF}$ of inserts was quite variable and may be due to various factors, including RHuA insert-specific properties (isolated cultures require $>4$ weeks to mature during which time cells/tissues may incur individuality) and small differences in ambient room temperature. Nonetheless, it appears digital dispensing that avoids higher volumes of DMSO may offer a solution enabling CBF measurements immediately postexposure.

\section{Summary}

While the use of digital dispensing to enable precise delivery of test materials has been available for some years, ${ }^{23}$ the use of these devices to deliver apical exposures to the apical airway compartment of RHuA is novel. Although smoke or aerosol generators can create an exposure that more closely resembles what occurs in vivo, no standardized method exists for determining dosimetry or for understanding the materials delivered to the airway surface. Hand pipetting is a reliable means by which to apply an exposure to an RHuA airway surface, but is not very physiological and has the beforementioned effects on rheology. Hand pipetting may also become complicated with high-capacity microwell plates that receive concentration arrays of different materials.

Seemingly a hybrid between the two other options mentioned, the D300 digital dispenser seems to fill a gap between these two methods by providing a means to precisely deliver a known amount of test material in DMSO to the airway surfaces of RHuA tissues. As a solvent, DMSO is widely used for materials not soluble in an aqueous environment. This includes many constituents found in TPM-a commonly used extract to evaluate the effects of tobacco smoke products. While the D300 was tested using DMSO-based dispensing, it also has the capability of dispensing aqueous solutions that contain a small amount of surfactant. Testing of this potentially less toxic alternate solution will highlight the D300's capability and may allow for a more aggressive repeat exposure testing paradigm when dispensing higher volumes.

The dispenser's user-friendly interface also allows the setup and seamless delivery of concentration arrays of test materials such as pharmaceuticals, environmental materials, or tobacco product-based e-cigarette constituents so that product screening should be easily conducted. This scenario would also benefit from higher capacity multiwell plates for RHuA tissues such as the 96-well plate. The coordinate-based dispensing for 96 wells would allow a higher number of test systems so that screening of test materials can be conducted faster in a more cost-effective manner, but while still using a more physiological 3D RHuA model such as MucilAir ${ }^{\mathrm{TM}}$.

\section{Conclusion}

Although this novel technology is still undergoing characterization, it has demonstrated promising results as a method by which materials (e.g., tobacco-based extracts) may be exposed into an ALI-based culture format and onto the apical surface of RHuA tissue. The increasing use of RHuA tissues for the testing of inhaled materials such as tobacco smoke or e-cigarette vapors (containing harmful or potentially harmful constituents) has faced challenges in determining the quantities of materials the tissue is exposed to. The precise delivery of known tobacco constituent concentrations in minute volumes to the apical surface of RHuA tissues may circumvent some of these dosimetry challenges and offer researchers an alternative exposure system that can be useful in appropriate experimental settings.

\section{Acknowledgments}

The authors would like to thank Drs. Rodger Curren and Mario Aragon (IIVS, Inc.) for the critical review of the manuscript and the following groups for their help, contributions, and support: Tecan (Simon Fogarty) and HP, Inc., (Christie Dudenhoefer and Dave Ochs) for the use of the D300 and support/interactions that led to its successful initial evaluation; BAT (Investments) Limited (Marianna Gaça and Linsey Haswell) and the UNC (Robert Tarran's Lab) for the kind contributions of TPM extract.

\section{Author Disclosure Statement}

IIVS, Inc., is a not-for-profit contract research organization that offers assays using in vitro and ex vivo models that may be exposed using the described D300 digital dispensing system. MucilAir tissues were created by Epithelix Sàrl and sent to IIVS for work to be conducted. The authors, S.C. and S.H., are employees/owners of Epithelix Sàrl.

\section{References}

1. Manuppello JR, Sullivan KM. Toxicity assessment of tobacco products in vitro. Altern Lab Anim 2015:43;39-67.

2. Adamson J, Haswell LE, Phillips G, et al. In vitro models of chronic obstructive pulmonary disease (COPD). In: Bronchitis. DI MartÃn-Loeches (ed.). Southampton, UK: British American Tobacco; 2011.

3. Behrsing H, Raabe H, Manuppello J, et al. Assessment of in vitro COPD models for tobacco regulatory science: Workshop proceedings, conclusions and paths forward for in vitro model use. Altern Lab Anim 2016:44; 129-166. 
4. BeruBe K, Aufderheide M, Breheny D, et al. In vitro models of inhalation toxicity and disease. The report of a FRAME workshop. Altern Lab Anim 2009:37;89-141.

5. Laschke MW, Menger MD. Life is 3D: Boosting spheroid function for tissue engineering. Trends Biotechnol 2016:35; 133-144.

6. Roomans GM. Tissue engineering and the use of stem/progenitor cells for airway epithelium repair. Eur Cell Mater 2010:19;284-299.

7. Johnson MD, Schilz J, Djordjevic MV, et al. Evaluation of in vitro assays for assessing the toxicity of cigarette smoke and smokeless tobacco. Cancer Epidemiol Biomarkers Prev 2009:18;3263-3304.

8. Iskandar AR, Xiang Y, Frentzel S, et al. Impact assessment of cigarette smoke exposure on organotypic bronchial epithelial tissue cultures: A comparison of mono-culture and coculture model containing fibroblasts. Toxicol Sci 2015: 147;207-221.

9. Kuehn D, Majeed S, Guedj E, et al. Impact assessment of repeated exposure of organotypic 3D bronchial and nasal tissue culture models to whole cigarette smoke. J Vis Exp 2015: $12 ; 96$.

10. Li X. In vitro toxicity testing of cigarette smoke based on the air-liquid interface exposure: A review. Toxicol In Vitro 2016:36;105-113.

11. Behrsing HP, Hill E, Raabe HA, et al. In vitro exposure systems and dosimetry assessment tools for inhaled tobacco products, 2016; Workshop Report.

12. Aufderheide M, Knebel JW, Ritter D. An improved in vitro model for testing the pulmonary toxicity of complex mixtures such as cigarette smoke. Exp Toxicol Pathol 2003:55;51-57.

13. Scian MJ, Oldham MJ, Kane DB, et al. Characterization of a whole smoke in vitro exposure system (Burghart Mimic Smoker-01). Inhal Toxicol 2009:21;234-243.

14. Thorne D, Adamson J. A review of in vitro cigarette smoke exposure systems. Exp Toxicol Pathol 2013:65;1183-1193.
15. Thorne D, Kilford J, Payne R, et al. Characterisation of a Vitrocell(R) VC 10 in vitro smoke exposure system using dose tools and biological analysis. Chem Cent J 2013:7;146.

16. Wright C. Standardized methods for the regulation of cigarettesmoke constituents. Trends Analyt Chem 2015:66;118-127.

17. Borgerding M, Klus H. Analysis of complex mixturesCigarette smoke. Exp Toxicol Pathol 2005:57 Suppl 1;43-73.

18. Fulcher ML, Gabriel S, Burns KA, et al. Well-differentiated human airway epithelial cell cultures. Methods Mol Med 2005:107;183-206.

19. Fulcher ML, Randell SH. Human nasal and tracheobronchial respiratory epithelial cell culture. Methods Mol Biol 2013:945;109-121.

20. Harvey PR, Tarran R, Garoff S, et al. Measurement of the airway surface liquid volume with simple light refraction microscopy. Am J Respir Cell Mol Biol 2011:45;592-599.

21. Haswell LE, Hewitt K, Thorne D, et al. Cigarette smoke total particulate matter increases mucous secreting cell numbers in vitro: A potential model of goblet cell hyperplasia. Toxicol In Vitro 2010:24;981-987.

22. Corcoran TE, Thomas KM, Brown S, et al. Liquid hyperabsorption as a cause of increased DTPA clearance in the cystic fibrosis airway. EJNMMI Res 2013:3;14.

23. Jones RE, Zheng W, McKew JC, et al. An alternative direct compound dispensing method using the HP D300 digital dispenser. J Lab Autom 2013:18;367-374.

Address correspondence to:

Dr. Holger P. Behrsing Respiratory Toxicology Program Institute for In Vitro Sciences, Inc. 30 W. Watkins Mill Road, Suite 100 Gaithersburg, MD 20878

E-mail: hbehrsing@iivs.org 